TITLE:

\title{
Energy-loss straggling caused by the inhomogeneity of target material
}

\author{
$\operatorname{AUTHOR}(\mathrm{S})$ : \\ Tosaki, Mitsuo
}

CITATION:

Tosaki, Mitsuo. Energy-loss straggling caused by the inhomogeneity of target material. JOURNAL OF APPLIED PHYSICS 2006, 99(3): 034905.

\section{ISSUE DATE:}

2006-02-01

URL:

http://hdl.handle.net/2433/50507

\section{RIGHT:}

Copyright 2006 American Institute of Physics. This article may be downloaded for personal use only. Any other use requires prior permission of the author and the American Institute of Physics. 


\title{
Energy-loss straggling caused by the inhomogeneity of target material
}

\author{
Mitsuo Tosaki ${ }^{a)}$ \\ Radioisotope Research Center, Kyoto University, Kyoto 606-8501, Japan
}

(Received 5 September 2005; accepted 16 December 2005; published online 14 February 2006)

\begin{abstract}
Rutherford backscattering spectroscopy (RBS) accompanying with the sharp 4.808-MeV resonance of proton beams in carbon has been applied to examine the energy-loss straggling in detail, which causes the broadening of proton beam energy in the penetrating path. In the present measurements, RBS peak profiles of homogeneous and inhomogeneous carbon materials have been obtained with an incident energy of $5.5 \mathrm{MeV}$. Careful analyses of those profiles have revealed that the energy-loss straggling can be separated into two parts; one is collision straggling and the other is density straggling. The collision straggling is caused by the statistical fluctuation in collisions of proton with target atoms, which has been intensively studied since the theoretical work by Bohr [Philos. Mag. 30, 581 (1915)]. The density straggling is caused by the statistical fluctuation in local density of target material, which has been discussed in the present work. The random inhomogeneity as a measure of the fluctuation in the local density is introduced into the existing theory of the energy-loss straggling. Following the theoretical treatment, we have successfully deduced the random inhomogeneity of various carbon materials and the spatial spread of 4.808-MeV resonance protons in the materials. Some applications of the present method for the RBS with the sharp resonance are also presented. (C) 2006 American Institute of Physics. [DOI: 10.1063/1.2168299]
\end{abstract}

\section{INTRODUCTION}

When monoenergetic charged particles penetrate the target materials of single element, the stopping power $S$, i.e., the energy loss per unit path length, is expressed as

$$
S=-Z N C,
$$

where $Z$ is the atomic number of the element and $N$ is the number of the atoms in unit volume. The quantity $C$ is the summation of $\sigma_{k} Q_{k}$ for all collisions in unit path length of the charged particle:

$$
C=\sum_{k} \sigma_{k} Q_{k},
$$

where $\sigma_{k}$ is the cross section of the $k$ th process in possible collisions of the charged particle with electrons in the target and $Q_{k}$ is the energy loss of the $k$ th collision process. The number $N$ in Eq. (1) is

$$
N=\frac{N_{A}}{A} d,
$$

where $N_{A}$ is the Avogadro number, i.e., $6.02 \times 10^{-23} / \mathrm{mol}$, and $A$ is the atomic weight of the element. Then, $S$ is given by

$$
S=-Z\left(N_{A} / A\right) d C .
$$

Since there are many channels for the collision and each collision randomly takes place through one of the possible channels, $C$ is treated as a stochastic variable. While the density of homogeneous materials is constant, that of inhomogeneous materials changes locally in the proton path. If the change in density is statistically random, $d$ in Eq. (4) can be treated as a stochastic variable. We call this randomness in

\footnotetext{
${ }^{a)}$ Electronic mail: tosaki@barium.rirc.kyoto-u.ac.jp
}

the density as "random inhomogeneity." It is noted that the bulk density of the target material is not the same as $d$ in Eq. (4); the bulk density is equal to the statistic average of $d$. Thus, the fluctuation of the stopping power $S$ comes from two origins; one is the collision straggling caused by $C$ and the other is the density straggling caused by $d$. Since the theoretical work by Bohr, ${ }^{1}$ there have been performed a lot of experimental and theoretical studies on the energy-loss straggling, limiting only to the fluctuation in collision processes. This phenomenon in various materials is now widely applied in many fields, ${ }^{2-4}$ i.e., material and medical sciences. However, there has been no investigation on the density straggling; this straggling was clearly overlooked in most of the previous studies.

For carbon materials, an experimental method possible to examine the density straggling is the Rutherford backscattering spectroscopy (RBS) with the ${ }^{12} \mathrm{C}(p, p){ }^{12} \mathrm{C}$ reaction, which accompanies a very sharp nuclear resonance at the proton energy of $4.808 \mathrm{MeV}$; a natural linewidth of this resonance is $12 \mathrm{keV}$ (see Table II in Ref. 5). An evident peak corresponding to the sharp resonance appears in the RBS energy spectrum, when proton energies are higher than the resonance energy, $4.808 \mathrm{MeV}$. The profile with the sharp peak is critically affected by the energy-loss straggling which causes the broadening of the peak width. The quantitative analysis of the width broadening for homogeneous and inhomogeneous materials makes possible to separate the density straggling from the energy-loss straggling.

Following the previous work, ${ }^{6}$ RBS measurements with homogenous and inhomogeneous carbon targets have been performed in the present work. With an aid of the theoretical treatment of energy-loss straggling presently extended, the random inhomogeneity as well as the spatial spread of protons resonantly scattered in the target materials have system- 


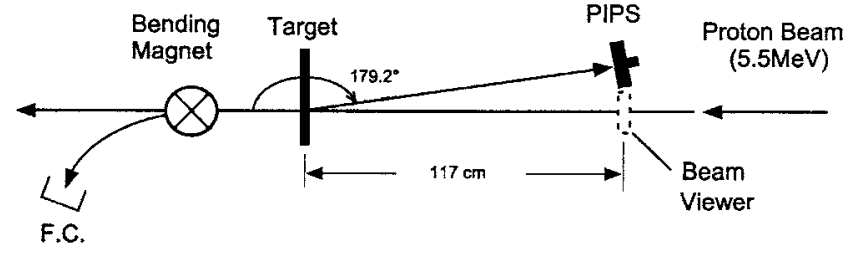

FIG. 1. Experimental setup for the resonant backscattering measurements: passivated implanted planar silicon (PIPS) detector, beam viewer, scintillator ceramic board used for beam-line alignment, bending magnet used for measurements of the excitation function to eliminate unwanted backscattering protons from beam dump, and Faraday cup (FC).

atically deduced from the observed peak profiles. The purpose of this work is to reveal experimentally the overlooked process of the energy-loss straggling, i.e., the density straggling, and to establish an experimental technique for determining the random inhomogeneity and the spatial spread.

\section{EXPERIMENT}

\section{A. Experimental setup}

The RBS measurements were performed with the 8MV tandem accelerator of the Department of Physics of Kyoto University. An incident proton beam of $5.5 \mathrm{MeV}$ was used with a stability less than $\pm 2.3 \mathrm{keV}$. The beam size on the target was 1-2 $\mathrm{mm}$ in diameter. The experimental method used in this study is the same as that described in the previous paper. ${ }^{6}$ The experimental setup is shown in Fig. 1; the scattering angle is $179.2^{\circ}$, the distance between the detector and the target is $117 \mathrm{~cm}$, and the solid angle, in which the beam point on the target faces to the detector, is estimated to be $1.83 \times 10^{-5} \mathrm{sr}$. This solid angle results in a very small energy spread for protons backscattered from the target materials. For example, the spread was estimated to be $0.064 \mathrm{keV}$ for the $3.427-\mathrm{MeV}$ protons, which are backscattered by the $4.808-\mathrm{MeV}$ nuclear resonance of $5.5-\mathrm{MeV}$ incident protons. The extreme angle in the backward direction and the long distance are essential to obtain better signal-tonoise $(\mathrm{S} / \mathrm{N})$ ratios in RBS spectra, i.e., the count ratio of the nuclear resonance scattering to Rutherford backscattering: typical $\mathrm{S} / \mathrm{N}$ values obtained with homogeneous targets are 0.58 at $138^{\circ}, 1.9$ at $166^{\circ}$, and 2.8 at $179.2^{\circ}$ (Ref. 7). Furthermore, this angle is helpful to avoid extra fluctuations caused by the surface roughness, because the incident protons enter in a direction normal to the target surface.

The backscattered protons were detected with a passivated implanted planar silicon (PIPS) detector with an active area of $25 \mathrm{~mm}^{2}$ and a thickness of $300 \mu \mathrm{m}$ : the nominal resolution is $11.0 \mathrm{keV}$ for $5.486-\mathrm{MeV} \alpha$ particles from Am241. The resolution of the PIPS in the present setup geometry was determined to be $14.4 \mathrm{keV}$ at the proton energy of $3.427 \mathrm{MeV}$ with the target thickness of $8.3 \mu \mathrm{g} / \mathrm{cm}^{2}$. It is noted that the resolution value contains the spread, $2.3 \mathrm{keV}$, coming from the fluctuation in the incident proton energy. The vacuum pressure was $1.3 \times 10^{-4} \mathrm{~Pa}$ during the measurements.

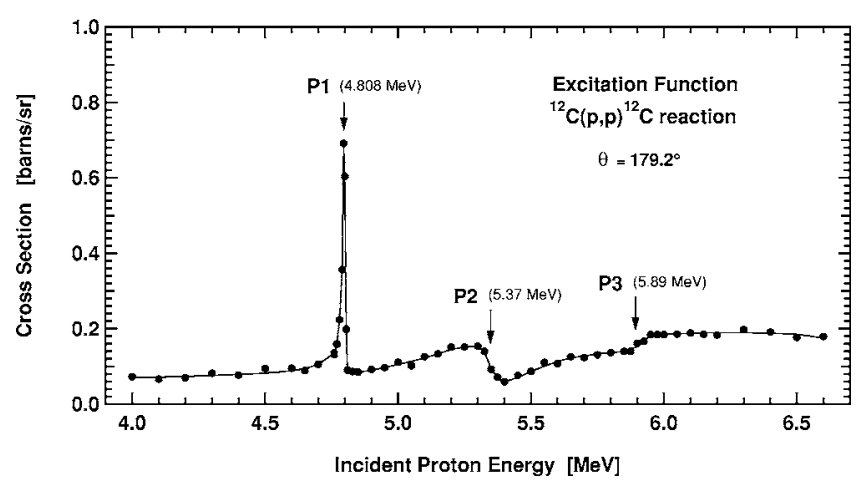

FIG. 2. Excitation function for ${ }^{12} \mathrm{C}(p, p){ }^{12} \mathrm{C}$ reaction measured at $179.2^{\circ}$ in laboratory. P1, P2, and P3 indicate the resonances at 4.808, 5.37, and $5.89 \mathrm{MeV}$, respectively.

\section{B. Targets}

We have used commercial available materials of crystal structure as homogeneous targets, i.e., highly oriented pyrolytic (HOP) graphite and chemical-vapor deposition (CVD) diamond with the densities of 2.26 and $3.52 \mathrm{~g} / \mathrm{cm}^{3}$, respectively. The thickness of these targets was $400-500 \mu \mathrm{m}$, which is over twice the range of the $5.5-\mathrm{MeV}$ proton in the target. These targets with crystal structure have a uniform density as well as a clean surface.

Three self-supporting targets with different densities were made of powdery carbons with different grain sizes: C-60 fullerenes for a fine powder $(\sim 1 \mathrm{~nm})$, graphite powder for a medium size $(\sim 1 \mu \mathrm{m})$, and charcoal powder for a coarse size $(\sim 100 \mu \mathrm{m})$. These powdery carbons mounted in an aluminum ring $(8 \mathrm{~mm}$ in inside diameter, $10 \mathrm{~mm}$ in outside diameter, and $3 \mathrm{~mm}$ in height) were pressed by 1-1.5 tons with a tableting machine. The bulk densities of the targets were $1.43,0.98$, and $0.63 \mathrm{~g} / \mathrm{cm}^{3}$ according to the grain sizes, which were determined by measuring the weight and the volume within an error of $2 \%$.

\section{OBSERVATION AND ANALYSES}

\section{A. Excitation function and RBS spectra}

The excitation function of protons for carbon, which was observed at the backward angle of $179.2^{\circ}$, is given in Fig. 2 . The peak P1 at the proton energy of $4.808 \mathrm{MeV}$ is so sharp that the yield at the resonance energy is about ten times greater than that at off-resonance energies; the natural linewidth is $12 \mathrm{keV}$. The yield at the off-resonance comes from Rutherford backscattering. The cross section of the nuclear resonance is larger with increasing the scattering angle up to $180^{\circ}$, while that of the Rutherford backscattering is smaller. There appear two other resonances labeled by P2 and P3 in Fig. 2; their resonance energies are 5.37 and $5.89 \mathrm{MeV}$ with the natural widths of $125 \mathrm{keV}$ (Ref. 5) and $75 \mathrm{keV},{ }^{8}$ respectively.

RBS spectra of HOP graphite and CVD diamond at the scattering angle of $179.2^{\circ}$ are shown in Fig. 3. Labels P1 and P2 in Fig. 3 indicate the peaks caused by the nuclear resonances at 4.808 and $5.37 \mathrm{MeV}$, respectively. It is noted that there is no difference between the two spectra in the whole energy ranges observed. Since HOP graphite and CVD dia- 


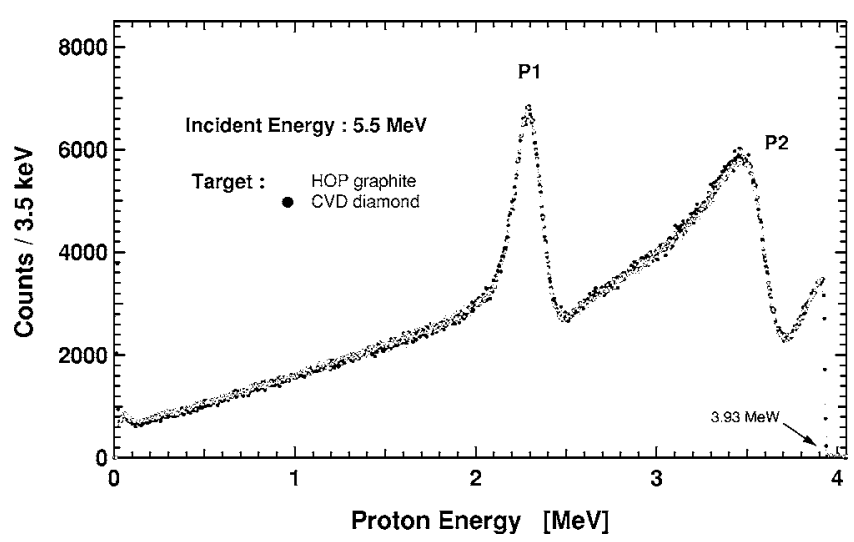

FIG. 3. Energy spectrum of backscattered protons by HOP graphite and CVD diamond targets with an incident proton energy of $5.5 \mathrm{MeV}$. P1 and $\mathrm{P} 2$ indicate the resonances corresponding to those in the excitation function of Fig. 2. The energy of surface scattering is around $3.93 \mathrm{MeV}$, as shown.

mond are pure crystal, the arrangement of carbon atoms inside the materials is expected to be completely homogeneous. The spectra in Fig. 3 show that the RBS spectrum does not depend on the density for homogeneous targets.

RBS spectra of the three samples made of powdery carbon with different bulk densities (1.43, 0.98, and $0.63 \mathrm{~g} / \mathrm{cm}^{3}$ ) are shown in Fig. 4. For comparison, the spectrum of HOP graphite $\left(2.26 \mathrm{~g} / \mathrm{cm}^{3}\right)$ is also shown in the figure. The P1 peak width for HOP graphite, i.e., homogeneous target, is the narrowest and the width is broader with decreasing the bulk density. It is clear that the samples of the powdery carbon are not as homogeneous as the crystals of HOP graphite and CVD diamond. The spectra in Fig. 4 show that the inhomogeneity of the target materials makes the peak width of the sharp resonance wider. On the contrary, the profiles of the P2, of which linewidth is much broader than that of $\mathrm{P} 1$ peak, do not depend on the target materials presently employed.

\section{B. Composition of peak width}

The resonance peak width $W_{R}$ is expressed by

$$
W_{R}=W_{N} \otimes W_{B} \otimes W_{D} \otimes W_{S} \otimes W_{E},
$$

where $W_{N}$ is the linewidth of the nuclear resonance peak (12 keV for $4.808-\mathrm{MeV}$ resonance), $W_{B}$ is the spread of incident beam $(\sim 2.3 \mathrm{keV}), W_{D}$ is the response width of detector $(\sim 14.4 \mathrm{keV}), W_{S}$ is the kinematics spread by the solid angle in the present experimental setup $(0.064 \mathrm{keV})$, and $W_{E}$ is the energy-loss straggling to be determined. The symbol $\otimes$ in Eq. (5) indicates that the method appropriate for the distribution function of each factor is to be employed for the composition. The notation $W$ indicates the full width at half maximum FWHM value corresponding to the distribution function of each factor. The descriptions in parentheses indicate the order of each width or spread in the present RBS measurement, as discussed in Sec. II A.

The shape of the resonance peak is composed of the symmetry sharp peak expressed by a Lorentzian and the asymmetry shape coming from the interference between the nuclear resonance and Rutherford backscattering. ${ }^{9}$ The resonance shape is broadened by the four factors, $W_{B}, W_{D}, W_{S}$,

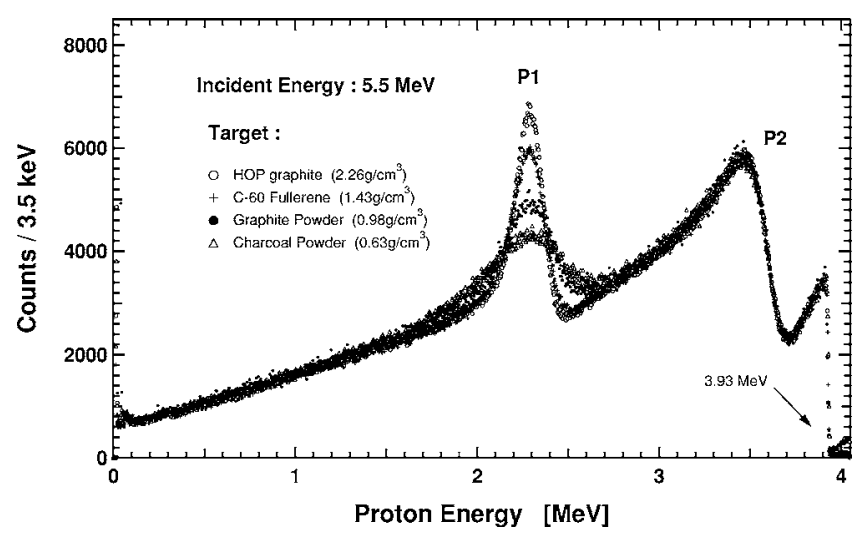

FIG. 4. Energy spectra of backscattered protons by C-60 fullerene, graphite powder, and charcoal powder targets with an incident proton energy of $5.5 \mathrm{MeV}$. A spectrum for the homogeneous material, i.e., HOP graphite, is shown as a reference. P1 and P2 indicate the resonances corresponding to those in the excitation function of Fig. 2. The energy of surface scattering is around $3.93 \mathrm{MeV}$, as shown.

and $W_{E}$ in Eq. (5), which are approximately expressed by Gaussian. So, the shape of the resonance peak observed is a result of the convolution of the Lorentzian, the asymmetry distribution of the interference, and the four Gaussians.

\section{C. $\chi^{2}$ fit for RBS spectra}

The deconvolution of the observed resonance shape has been performed by the technique of $\chi^{2}$ fit. ${ }^{10}$ The shape of the symmetry resonance peak is given by Voigt profile, ${ }^{11}$ i.e., the convolution of Lorentzian and Gaussian:

$$
\begin{aligned}
V(x ; & \left.W_{N}, W_{G}, x_{p}\right) \\
= & \frac{2 \ln 2}{\pi^{3 / 2}}\left(\frac{W_{N}}{W_{G}^{2}}\right) \\
& \quad \times \int_{0}^{\infty} \frac{\exp \left(-t^{2}\right) d t}{\left[t-2 \sqrt{\ln 2}\left(x-x_{p}\right) / W_{G}\right]^{2}+\ln 2\left(W_{N} / W_{G}\right)^{2}},
\end{aligned}
$$

where $W_{N}$ and $W_{G}$ are FWHMs of Lorentzian and Gaussian shapes, respectively, and $x_{p}$ is the energy at the peak position. This function is normalized as

$$
\int_{-\infty}^{\infty} V\left(x ; W_{N}, W_{G}, x_{p}\right) d x=1 .
$$

The shape of the asymmetry caused by the interference term is given by

$$
\begin{aligned}
& I\left(x ; W_{N}, W_{G}, x_{p}\right) \\
& =\frac{2 \ln 2}{\pi^{3 / 2}}\left(\frac{W_{N}}{W_{G}^{2}}\right) \\
& \quad \times \int_{0}^{\infty} \frac{\exp \left(-t^{2}\right)\left[t-2 \sqrt{\ln 2}\left(x-x_{p}\right) / W_{G}\right] d t}{\left[t-2 \sqrt{\ln 2}\left(x-x_{p}\right) / W_{G}\right]^{2}+\ln 2\left(W_{N} / W_{G}\right)^{2}} .
\end{aligned}
$$

Then, the model function of the RBS spectrum is given by 


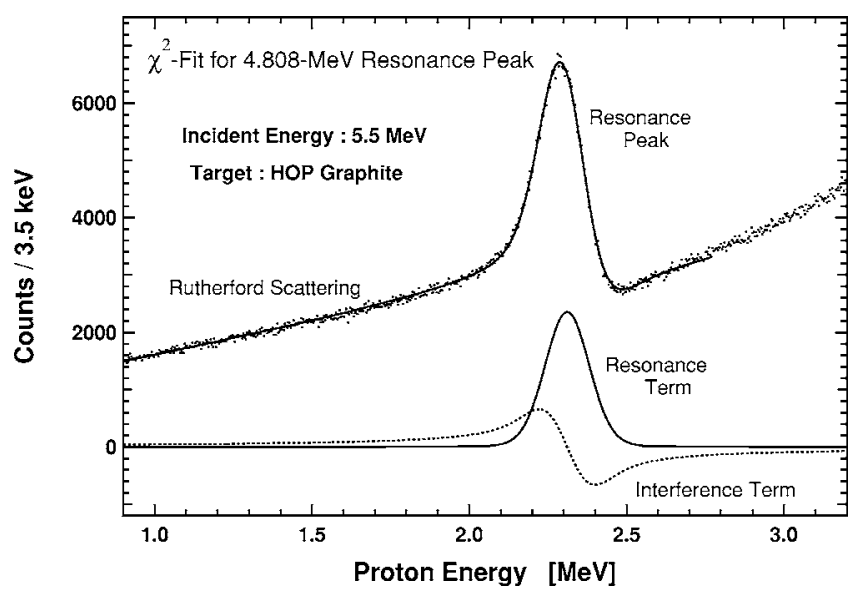

FIG. 5. The measured energy spectra for 5.5-MeV proton bombardment of the HOP graphite target and the curve-fit result for the $4.808-\mathrm{MeV}$ resonance peak. The solid and dotted curves at the bottom indicate the resonance term and the interference term, respectively.

$$
\begin{aligned}
F\left(x ; W_{N}, W_{G}, x_{p}, A, B, a, b\right)= & (a+b x)+A V\left(x ; W_{N}, W_{G}, x_{p}\right) \\
& +B I\left(x ; W_{N}, W_{G}, x_{p}\right),
\end{aligned}
$$

where $A$ and $B$ are the intensities of the peak and the interference term, respectively. The first term is the straight line to express the background continuum.

The adjustable parameters in Eq. (9), i.e., $W_{N}, W_{G}, x_{p}, A$, $B, a$, and $b$, can be determined by the $\chi^{2}$ fit of the observed RBS spectra. The typical results of the $\chi^{2}$ fit are shown in Fig. 5. The values of $W_{N}, W_{G}, x_{p}, A$, and $B$, and their errors determined for HOP graphite and CVD diamond, are listed in Table I. It is noted that the width $W_{G}$ is the convolution of the four factors, i.e., $W_{B} \otimes W_{D} \otimes W_{S} \otimes W_{E}$, and is given by

$$
W_{G}=\sqrt{W_{B}^{2}+W_{D}^{2}+W_{S}^{2}+W_{E}^{2}} \cong W_{E} .
$$

As shown in Table I, $W_{G}$ is $\sim 160 \mathrm{keV}$, which is much larger than $W_{B}(\sim 2.3 \mathrm{keV}), W_{D}(\sim 14.4 \mathrm{keV})$, and $W_{S}(0.064 \mathrm{keV})$. This means that the energy-loss straggling $W_{E}$ is approximately equal to $W_{G}$, as expressed in Eq. (10).

\section{THEORETICAL TREATMENT OF DENSITY STRAGGLING}

\section{A. Energy loss}

In the present RBS measurements, the incident protons lose their energy in the incoming path from the surface to the position where the resonance takes place as well as the outgoing path from the resonance point to the surface. The energy loss in the incoming path $E_{L 1}$ is

TABLE I. The adjustable parameters of $W_{N}, W_{G}, x_{p}, A$, and $B$ in $\chi^{2}$ fit, and their errors.

\begin{tabular}{lccccc}
\hline \hline & $\begin{array}{c}W_{N} \\
(\mathrm{keV})\end{array}$ & $\begin{array}{c}W_{G} \\
(\mathrm{keV})\end{array}$ & $\begin{array}{c}x_{p} \\
(\mathrm{keV})\end{array}$ & $A$ & $B$ \\
\hline CVD diamond & $14 \pm 4$ & $161.3 \pm 2.8$ & $2311.0 \pm 0.7$ & $65.6 \pm 8.6$ & $-6.4 \pm 2.1$ \\
HOP graphite & $13 \pm 4$ & $158.2 \pm 2.4$ & $2311.4 \pm 0.6$ & $639.6 \pm 7.3$ & $-6.6 \pm 1.9$ \\
Average & $13 \pm 3$ & $159.5 \pm 1.8$ & $2311.2 \pm 0.5$ & $645.6 \pm 5.6$ & $-6.5 \pm 1.4$ \\
\hline \hline
\end{tabular}

TABLE II. The energy loss in the incoming path and that in the outgoing path.

\begin{tabular}{cccccc}
\hline \hline Peak & $\begin{array}{c}E_{2}^{\mathrm{a}} \\
(\mathrm{keV})\end{array}$ & $\begin{array}{c}W_{N}^{\mathrm{a}} \\
(\mathrm{keV})\end{array}$ & $\begin{array}{c}E_{L 1} \\
(\mathrm{keV})\end{array}$ & $\begin{array}{c}E_{L 2} \\
(\mathrm{keV})\end{array}$ & $\begin{array}{c}E_{L}\left(=E_{L 1}+E_{L 2}\right) \\
(\mathrm{keV})\end{array}$ \\
\hline P1 & 4808 & 12 & 692 & 1122 & 1814 \\
$\mathrm{P} 2$ & 5370 & 125 & 130 & 334 & 464 \\
\hline \hline
\end{tabular}

${ }^{\mathrm{a}}$ Reference 5 .

$$
E_{L 1}=E_{1}-E_{2},
$$

where $E_{1}$ is the incident energy of proton and $E_{2}$ is the resonance energy. The energy loss in the outgoing path $E_{L 2}$ is estimated as

$$
E_{L 2}=\kappa E_{2}-E_{4},
$$

where $E_{4}$ is the energy of the resonance peak in the RBS spectra. The factor $\kappa$ comes from the kinematics of the ${ }^{12} \mathrm{C}(p, p){ }^{12} \mathrm{C}$ reaction, which is given by

$$
\kappa=\left(\frac{m_{p} \cos \theta+\sqrt{m_{N}{ }^{2}-m_{p}{ }^{2} \sin ^{2} \theta}}{m_{N}+m_{p}}\right)^{2},
$$

where $m_{N}$ and $m_{p}$ are the masses of carbon atom and proton, respectively, and $\theta$ is the scattering angle. The factor $\kappa$ for the present experimental setup of $\theta=179.2^{\circ}$ is estimated to be 0.714 .

All peaks in Figs. 3 and 4 are at the same position, indicating that the energy loss in the present RBS measurements does not depend on the target materials; $E_{4}$ is $2311 \mathrm{keV}$ for the P1 peak and $3450 \mathrm{keV}$ for the P2 peak. For the peaks $\mathrm{P} 1$ and $\mathrm{P} 2$, the energy losses $E_{L 1}$ and $E_{L 2}$ estimated from Eqs. (11) and (12) are listed in Table II with the resonance energy $E_{2}$ and its natural width $W_{N}$. As seen in this table, the total energy loss for the $\mathrm{P} 1$ peak, $E_{L}\left(=E_{L 1}+E_{L 2}\right)$, is about 150 times larger than $W_{N}$, while $E_{L}$ for the P2 peak is of the same order of $W_{N}$. This is a main reason why the difference between homogeneous and inhomogeneous materials cannot be observed in $\mathrm{P} 2$ peaks of the RBS spectra; the width $W_{N}$ for the $\mathrm{P} 2$ peak is too broad compared with the total energy loss $E_{L}$.

\section{B. Bohr's energy-loss straggling}

According to the theoretical treatment by Bohr, ${ }^{1}$ the collision straggling does not depend on the energy of penetrating charged particles, except energies lower than several $\mathrm{keV}$. Then, the collision straggling in the expression of FWHM in the unit of $\mathrm{keV}$ is given by

$$
W_{C}=2.355 \Delta B \sqrt{d X},
$$

where $X$ is the penetration depth and $d$ is the density of the target material defined. The factor $\Delta B$ is

$$
\Delta B=\sqrt{4 \pi e^{4} Z_{1}^{2} Z_{2} \frac{N_{A}}{A}},
$$

where $Z_{1}$ is the atomic number of the projectile and $Z_{2}$ is that of the target. The constant 2.355 is the conversion factor from the standard deviation to the FWHM in the Gaussian distribution. The factor $\Delta B$ for the collision straggling of 
proton in carbon is 2.80, when $X$ and $d$ are given in the units of $\mu \mathrm{m}$ and $\mathrm{g} / \mathrm{cm}^{3}$, respectively. The energy loss $E_{L}$ is given by

$$
E_{L}=-\int_{x_{1}}^{x_{2}} S d x
$$

where $S$ is the stopping power defined by Eq. (4), and $x_{1}$ and $x_{2}$ are two distances of protons from the surface of the target. When the penetration depth $X$ is sufficiently small, the energy loss in the depth $E_{L}$ is deduced as

$$
E_{L}=-\int_{0}^{X} S d x \approx Z\left(N_{A} / A\right) C(d X) .
$$

According to Eq. (14), the relation between $W_{C}$ and $\sqrt{E_{L}}$ is given as

$$
W_{C} \propto \sqrt{E_{L}} .
$$

In the RBS spectra, $W_{C}$ and $E_{L}$ correspond to the width and the position of the resonance peak in the RBS profiles, respectively. Relation (18) indicates that, in the collision straggling by Bohr, the resonance peak width does not change for the same energy loss in any materials with different densities. As seen in Fig. 3, the profiles of HOP graphite and CVD diamond are precisely the same as expected; these targets are completely homogeneous and the energy-loss straggling comes only from the fluctuation of the collision process, i.e., collision straggling. On the other hand, it is clearly shown in Fig. 4 that the peak widths of the inhomogeneous targets are broader than those of the homogeneous targets. This extra broadening of the peak width is caused by the fluctuation of the density of the target materials, i.e., are density straggling as explained below.

\section{Definition of random inhomogeneity}

The fluctuation in the energy loss $\delta E_{L}$ can be deduced from Eq. (16). When the variables $d$ and $C$ in Eq. (4) are assumed to be statistical, we obtain

$$
\frac{\delta E_{L}}{E_{L}}=\frac{\delta E_{C}}{E_{L}}+\frac{\delta d}{d},
$$

where $\delta d$ is the fluctuation in $d$ and $\delta E_{C}$ is that in the energy loss caused by the variable $C$ in Eq. (4). Further assuming that the distribution for $\delta d$ and $\delta E_{C}$ is given by Gaussian, we obtained

$$
\left(\Delta E_{L}\right)^{2}=\left(\frac{\Delta d}{d}\right)^{2} E_{L}^{2}+\left(\Delta E_{C}\right)^{2},
$$

for the standard deviation of the variable $\delta E_{L} ; \Delta E_{L}, \Delta E_{C}$, and $\Delta d$ are the energy-loss straggling, the collision straggling, and the density straggling in the expression of the standard deviation, respectively. It is noted that the FWHM values of those stragglings stand for 2.355 times the corresponding standard deviation.

\section{Spatial distribution in target materials}

Figure 6 illustrates the relation between the energy and penetration depth of protons in the RBS measurements. In

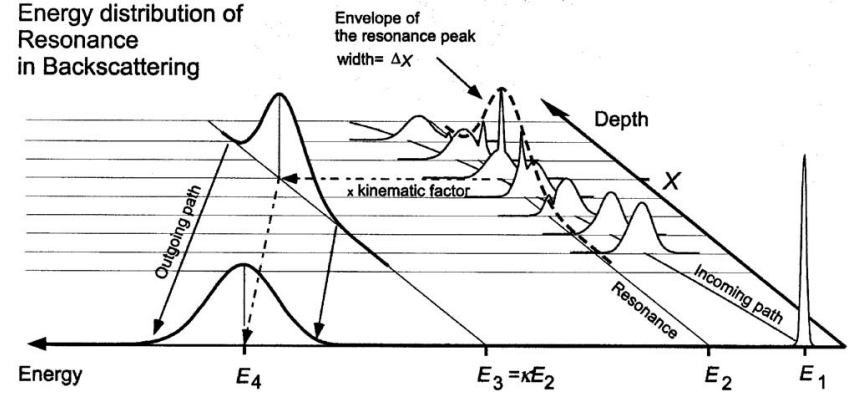

FIG. 6. A schematic illustration of the energy distribution around the resonance with the relation between the energy straggling and the spatial spread for penetrated proton beams. The dotted curve indicates the envelope curve of the peak points of the resonance, which indicates the spatial distribution of protons with the resonance energy in the target: $E_{1}$, incident energy of $5.5 \mathrm{MeV} ; E_{2}$, the resonance energy of $4.808 \mathrm{MeV} ; E_{3}$, the energy just after the scattering; $\kappa$, kinematic factor $0.714 ; E_{4}$, energy of outgoing protons at the surface; $X$, mean resonance depth; and $\Delta X$, depth straggling for the mean resonance depth.

the incoming path, the energy-loss straggling, i.e., the width of the energy distribution, increases with larger penetration depth. When the energy distribution contains the energy of the sharp resonance, the yield at the resonance energy is strongly enhanced. Then, the energy distribution has two components: the sharp peak as the $4.808-\mathrm{MeV}$ resonance and the broadening distribution as a result of the energy-loss straggling. The dotted curve shows an envelope that traces the top positions of the resonance peak. The curve means a spatial distribution of penetrating protons resonantly scattered with the energy of $4.808 \mathrm{MeV}$. As shown in Fig. 6, the observed width of the resonance peak at the energy of $E_{4}$ is affected by the spatial distribution of $4.808-\mathrm{MeV}$ protons created in the incoming path and the energy-loss straggling for the outgoing path length. Therefore, using the observed resonance peak and existing data of stopping power, we can estimate the spatial distribution at the penetration depth where the sharp resonance occurs.

The penetration depth $X$ from the surface to the resonance point is

$$
X=-\int_{E_{1}}^{E_{2}} \frac{d E}{S(E)}
$$

where $E_{1}$ and $E_{2}$ are initial and final energies of protons in the incoming path, respectively, and $S(E)$ is the stopping power as a function of the proton energy $E$. Energies $E_{1}$ and $E_{2}$ are fixed without fluctuations, i.e., $E_{1}=5.5 \mathrm{MeV}$ and $E_{2}$ $=4.808 \mathrm{MeV}$. Therefore, the fluctuation in $X$ comes from that in $S$. Then, the fluctuation $\delta X$ at the energy $E_{2}$ is expressed as

$$
\delta X=\delta S \int_{E_{1}}^{E_{2}} \frac{d E}{S^{2}(E)} .
$$

In this derivation, it is assumed that the fluctuation of the stopping power $\delta S$ does not depend on the energy of protons, according to the theoretical treatment by Bohr. ${ }^{1}$ Then, we obtain the following equation for the standard deviation of $\delta X:$ 
TABLE III. The energy-loss straggling, the random inhomogeneity, the spatial spread, and the penetration depth.

\begin{tabular}{lccccc}
\hline \hline & $\begin{array}{c}d \\
\left(\mathrm{~g} / \mathrm{cm}^{3}\right)\end{array}$ & $\begin{array}{c}W_{G} \\
(\mathrm{keV})\end{array}$ & $\begin{array}{c}X \\
(\mu \mathrm{m})\end{array}$ & $\begin{array}{c}W_{x}^{\mathrm{a}} \\
(\mu \mathrm{m})\end{array}$ & $\begin{array}{c}W_{d} / d^{\mathrm{b}} \\
(\%)\end{array}$ \\
\hline CVD diamond & $3.52 \pm 0.01$ & $159.5 \pm 1.8$ & $28.8 \pm 0.3$ & $3.1 \pm 0.1$ & 0 \\
HOP graphite & $2.26 \pm 0.01$ & $159.5 \pm 1.8$ & $44.8 \pm 0.5$ & $4.8 \pm 0.1$ & 0 \\
C-60 & $1.48 \pm 0.03$ & $191.4 \pm 1.0$ & $68.4 \pm 1.4$ & $8.4 \pm 0.2$ & $6.2 \pm 0.1$ \\
Graphite & $0.98 \pm 0.02$ & $301.6 \pm 3.1$ & $103.3 \pm 2.1$ & $18.9 \pm 0.3$ & $15.0 \pm 0.2$ \\
Charcoal & $0.63 \pm 0.01$ & $454.2 \pm 6.0$ & $160.8 \pm 3.2$ & $43.3 \pm 0.7$ & $25.0 \pm 0.4$ \\
\hline \hline
\end{tabular}

${ }^{\mathrm{a}} W_{X}=2.355 \Delta x$.

${ }^{\mathrm{b}} W_{d} / d=2.355(\Delta d / d)$.

$$
\begin{aligned}
(\Delta X)^{2} & =\left(\Delta E_{L 1}\right)^{2} R^{2}\left(E_{1}, E_{2}\right) \\
& =\left[\left(\frac{\Delta d}{d}\right)^{2} E_{L 1}{ }^{2}+\left(\Delta E_{C}\right)^{2}\right] R^{2}\left(E_{1}, E_{2}\right),
\end{aligned}
$$

where $\Delta E_{L 1}$ is the energy-loss straggling at the energy $E_{2}$, which is given by Eq. (20), and $R\left(E_{1}, E_{2}\right)$ is given by

$$
R\left(E_{1}, E_{2}\right) \equiv \frac{\int_{E_{1}}^{E_{2}} d E / S^{2}(E)}{\int_{E_{1}}^{E_{2}} d E / S(E)} .
$$

As explained before, the broadening of the resonance peak at the energy $E_{4}$ results from two origins. One is the spatial spread of 4.808-MeV protons as shown in Fig. 6 in the incoming path $\left(E_{1} \rightarrow E_{2}\right)$. The other is the energy-loss straggling in the outgoing path $\left(E_{3} \rightarrow E_{4}\right)$. Note that $E_{3}$ is the proton energy just after the resonance scattering, which is lower than the resonance energy by the amount of the kinematics of the scattering. The energy $E_{4}$ is given by

$$
E_{4}=E_{3}-\int_{0}^{X} S d x
$$

Then, the fluctuation $\delta E_{4}$ is deduced as

$$
\begin{aligned}
\delta E_{4} & =-\int_{0}^{\delta X} S d x-\int_{0}^{X}(\delta S) d x \\
& =\delta X S\left(E_{4}\right)-\delta E_{L 2} .
\end{aligned}
$$

Then, we obtain the following equation for the standard deviation $\Delta E_{4}$, i.e., the energy spread of proton outgoing from the target:

$$
\left(\Delta E_{4}\right)^{2}=\left[\Delta X S\left(E_{4}\right)\right]^{2}+\left(\Delta E_{L 2}\right)^{2},
$$

where the first term is the contribution of the spatial spread of $4.808-\mathrm{MeV}$ proton in the incoming path and the second term is that of the energy-loss straggling in the outgoing path. Substituting Eqs. (20) and (23) into Eq. (27), $\Delta E_{4}$ is expressed as

$$
\begin{aligned}
\left(\Delta E_{4}\right)^{2}= & \left(\frac{\Delta d}{d}\right)^{2}\left[E_{L 2}^{2}+S^{2}\left(E_{4}\right) E_{L 1}^{2} R^{2}\left(E_{1}, E_{2}\right)\right] \\
& +\left(\Delta E_{C}\right)^{2}\left[1+S^{2}\left(E_{4}\right) R^{2}\left(E_{1}, E_{2}\right)\right] .
\end{aligned}
$$

In the case of the homogeneous target, the first term of Eq. (28) is eliminated. So Bohr's energy-loss straggling at the depth of $X$ is obtained by

$$
\Delta E_{C}=\sqrt{\frac{\left(\Delta E_{4}\right)_{\text {homo }}^{2}}{1+S^{2}\left(E_{4}\right) R^{2}\left(E_{1}, E_{2}\right)}},
$$

where $\left(\Delta E_{4}\right)_{\text {homo }}$ is the observed resonance peak width for the homogeneous target. Using Eq. (27) in which the energyloss straggling for the inhomogeneous target stands for $\left(\Delta E_{4}\right)_{\text {inhomo }}$, we obtain the random inhomogeneity

$$
\frac{\Delta d}{d}=\sqrt{\frac{\left(\Delta E_{4}\right)_{\text {inhomo }}^{2}-\left(\Delta E_{4}\right)_{\text {homo }}^{2}}{E_{L 2}{ }^{2}+S^{2}\left(E_{4}\right) E_{L 1}{ }^{2} R^{2}\left(E_{1}, E_{2}\right)}} .
$$

Therefore, the spatial spread $\Delta X$, Bohr's energy-loss straggling $\Delta E_{C}$, and the random inhomogeneity $\Delta d / d$ have been estimated from Eqs. (23), (29), and (30), respectively. In this estimation, the stopping power $S(E)$ was quoted from the values calculated by Andersen and Ziegler ${ }^{12}$ and the energy spread $\Delta E_{4}$ was deduced from the Gaussian width $W_{G}$ by the deconvolution explained in Sec. III C: $\Delta E_{4}=W_{G} / 2.355$. As the stopping powers in Ref. 12 are defined with the carbon density of $2.26 \mathrm{~g} / \mathrm{cm}^{3}$, the $S(E)$ for the density of $d\left(\mathrm{~g} / \mathrm{cm}^{3}\right)$ is given by

$$
S(E)=\left(\frac{d}{2.26}\right) S_{Z}(E),
$$

where $S_{Z}(E)$ is the stopping power of carbon in Ref. 12. The results of the energy spread $W_{G}$, the penetration depth $X$, the spatial spread $W_{X}(=2.355 \Delta X)$, and the random inhomogeneity $W_{d} / d(=2.355 \Delta d / d)$ are listed in Table III.

\section{DISCUSSIONS}

The removal of the Lorentzian natural line shape and the asymmetric interference from the peak profile of the 4.808$\mathrm{MeV}$ resonance is necessary to examine quantitatively the Gaussian peak broadening caused by the energy-loss straggling. The $\chi^{2}$ fit is inevitable for this removal, as shown in Sec. III C. The natural linewidth $W_{N}$ presently determined by the fit is $13 \pm 3 \mathrm{keV}$, as shown in Table I. This is consistent with the value of $12 \mathrm{keV}$, which was obtained with the different experimental method in the previous work. ${ }^{5}$ The method of $\chi^{2}$ fit for the peak shape with the interference term is given elsewhere. ${ }^{13}$ 
The collision straggling $W_{C}$ which equals to 2.355 times the value deduced from Eq. (29), is $75.7 \pm 1.5 \mathrm{keV}$ for both homogeneous materials, i.e., HOP graphite and CVD diamond. With this value, the factor $\Delta B$ in Eq. (14) is estimated to be $3.2 \pm 0.1$; the penetration depth $X$ estimated from Eq. (21) is used in this derivation. The factor $\Delta B$ presently obtained is clearly larger than the theoretical value by Bohr, 2.80 (Ref. 1). The origin of this difference is not clear in the present situation. The one-dimensional Gaussian has been employed in the present analysis for the peak broadening caused by the energy-loss straggling. The improved analysis with a more reliable distribution, i.e., the three-dimensional Gaussian, is now in progress.

One of the typical features of the present RBS measurement is that the energy distribution at the $4.808-\mathrm{MeV}$ resonance is restricted to the region with a sharp width of $12 \mathrm{keV}$, even if the proton energies are much broadened by deep penetration in the target material. The energy-loss straggling in the incoming path results in the emission of 4.808$\mathrm{MeV}$ resonantly scattered protons, of which the position is spread around the penetration depth $X$ given by Eq. (21). These spatially spread protons give rise to the energy-loss straggling in the outgoing path from the point of the nuclear resonance to the surface of the target. Therefore, the observed broadening profile of the resonance peak in the RBS is the convolution of the spatial spread of the $4.808-\mathrm{MeV}$ proton at the last stage of the incoming path and the energyloss straggling of those resonantly scattered protons in the outgoing path, as explained by Eq. (27). By instituting Eq. (30) into Eq. (23), we can explicitly deduce the spatial spread of the $4.808-\mathrm{MeV}$ protons. Thus, the present experimental method is an excellent tool to obtain the beam spread inside the matter; such an information cannot be obtained with any other measurements.

The difference in the peak width $W_{G}$ for inhomogeneous targets is quantitatively related to the random inhomogeneity $W_{d} / d$, as listed in Table III. In this table, the $W_{d} / d$ values for CVD diamond and HOP graphite are set to zero within the statistical precision of the $W_{G}$ value presently measured, i.e., about $3 \mathrm{keV}$ as seen in Table II. The $W_{G}$ value for C-60 is $6.2 \% \pm 0.1 \%$ corresponding to the value of about $30 \mathrm{keV}$, which is the difference between the $W_{G}$ value of C-60 and that of the homogeneous materials, as seen in Table III. This indicates that the $W_{d} / d$ value can be determined within the precision of about $0.6 \%$ in the present RBS measurement. Because of this good sensitivity for the random inhomogeneity, the present RBS method may be extended to measure the energy-loss straggling of chemical compounds and mixtures with comparatively low concentrations of carbon.

In most of the ion-beam analyses with very thin foils, in which the projectiles pass through the target foils, the energy-loss straggling $W_{E}$ is usually attributed to the surface roughness defined by the fluctuation in foil thickness $W_{T}$ :

$$
W_{E}=S W_{T},
$$

where $S$ is the stopping power. If the fluctuation in thickness is assumed to be statistical, the surface roughness can be included in the random inhomogeneity of the foil target. Then, the energy-loss straggling in the thin foil is expressed by the convolution of the collision straggling and the density straggling including the surface roughness, of which expression is given by Eq. (20). When the material of the thin foil is homogeneous, $W_{d} / d$ in Eq. (20) equals to the surface roughness. Thus, the quantity $W_{d} / d$ can be explained as a measure of the surface roughness of thin crystal foils.

In the present work, the targets with random inhomogeneity have been successfully prepared by pressing powdery carbon. It is noted that the random inhomogeneity deduced in this work is inherent in the grain of each powdery carbon; the random inhomogeneity does not depend on the order of high pressing as long as the grain is not destroyed by the high pressure. Therefore, the bulk density of powdery carbon has no connection with the random inhomogeneity and also the broadening of the resonance peak on RBS.

The channeling phenomena are usually observed in the scattering of proton with crystal materials, i.e., CVD diamond and HOP graphite, while such phenomena do not occur in the scattering with inhomogeneous materials. As seen in Figs. 3 and 4, the spectrum shape at the energy of the surface scattering (around $3.93 \mathrm{MeV}$ ) is much the same for all the target materials, homogeneous and inhomogeneous; there does not appear any disorder caused by the channeling in the spectra for the homogeneous targets. It is concluded that the channeling phenomena do not occur in the present experimental setup for RBS.

Carbon is an attractive material having many allotropes such as amorphous, graphite, diamond, fullerene, and nanotube, which recently draw a lot of attention as device materials. The method for evaluating the random inhomogeneity in the present work is available for the diagnosis of packing fine particles of those materials in nanotechnology; there are some theoretical treatments on the packing but few experimental investigations. ${ }^{14}$ The method is also available for checking the degree of homogeneity in material science on compounds and mixtures of carbon. Furthermore, the spatial distribution of projectiles in the material, as deduced in Sec. IV C, is of interest on medical physics such as cancer therapy with ion beams. ${ }^{15}$ Thus, the present RBS with the sharp resonance may be directly applied to many types of measurements in the ion-beam analysis.

\section{CONCLUDING REMARKS}

The Rutherford backscattering spectroscopy (RBS) with the ${ }^{12} \mathrm{C}(p, p){ }^{12} \mathrm{C}$ reaction at the resonance energy of $4.808 \mathrm{MeV}$ has been applied to examine the energy-loss straggling of carbon materials. The extraction of the resonance peak has been performed by the $\chi^{2}$ fit with the model function consisting of Lorentzian natural line shape, asymmetric interference term, and Gaussian straggling shape. It has been revealed through the careful analyses of the fit that the energy-loss straggling in inhomogeneous materials is the superposition of the collision straggling approved in general and the density straggling so far overlooked. The density straggling has been built in the theoretical treatment for the energy-loss straggling, in which a parameter "random inhomogeneity" is defined as a measure to express the statistical fluctuation in local density. The present work has also shown 
that the spatial spread of resonantly scattered projectiles in the target materials can be observed by the RBS with the nuclear resonance reaction. The random inhomogeneity and the spatial spread can be directly deduced from the observed RBS profiles with the help of the theoretical treatment presently extended. The present experimental method for the energy-loss straggling is a powerful tool to investigate the inner structure of the target materials of carbon.

The present method to examine the energy-loss straggling is available only for the target materials of carbon because of the applied resonance reaction, i.e., ${ }^{12} \mathrm{C}(p, p){ }^{12} \mathrm{C}$ at the energy of $4.808 \mathrm{MeV}$. It is noted that there are many other useful nuclear resonance reactions of proton or $\alpha$ particles with light atoms such as nitrogen and oxygen. ${ }^{2}$ By employing such reaction, the present method may be extended to the analyses for other light elements.

\section{ACKNOWLEDGMENT}

I am very grateful to Professor Y. Isozumi for initiating this work, useful discussions, and valuable suggestions.
${ }^{1}$ N. Bohr, Mat. Fys. Medd. K. Dan. Vidensk. Selsk. 18, 8 (1948).

${ }^{2}$ Handbook of Modern Ion Beam Materials Analysis, edited by J. R. Tesmer and M. Nastasi (Materials Research Society, Pittsburgh, 1995).

${ }^{3}$ ICRU Report No. 59 (International Commission on Radiation Units and Measurements, Bethesda, MD, 1998).

${ }^{4}$ Proceedings of the 16th International Conference on Ion Beam Analysis, Albuquerque, NM, 2003, edited by G. Vizkelethy, F. D. McDaniel, S. Thevuthasan, and J. R. Tesmer [Nucl. Instrum. Methods Phys. Res. B 219-220, 1 (2004)].

${ }^{5}$ C. W. Reich, G. C. Phillips, and J. L. Russell, Jr., Phys. Rev. 104, 143 (1956).

${ }^{6}$ M. Tosaki, S. Ito, and N. Maeda, Nucl. Instrum. Methods Phys. Res. B 168, 543 (2000).

${ }^{7}$ M. Tosaki, S. Ito, and N. Maeda, Annual Report No. 1995 (Tandem V. G. Laboratory, Kyoto University, Japan).

${ }^{8}$ N. Nikolic, L. J. Lidofsky, and T. H. Kruse, Phys. Rev. 132, 2212 (1963).

${ }^{9}$ R. R. Roy and B. P. Nigam, Nuclear physics: Theory and Experiment (Wiley, New York, 1967).

${ }^{10}$ Y. Isozumi, Nucl. Instrum. Methods Phys. Res. A 235, 164 (1985).

${ }^{11}$ F. Schreier, J. Quant. Spectrosc. Radiat. Transf. 48, 743 (1992).

${ }^{12}$ H. H. Andersen and J. F. Ziegler, The Stopping and Ranges of Ions in Matter (Pergamon, New York, 1977), Vol. 3.

${ }^{13} \mathrm{M}$. Tosaki and Y. Isozumi, Nucl. Instrum. Methods Phys. Res. A (to be published).

${ }^{14}$ J. P. Stoquert and T. Szörényi, Phys. Rev. B 66, 144108 (2002).

${ }^{15}$ The Physics of Highly and Multiply Charged Ions, edited by F. J. Currel (Kluwer, Dordrecht, 2002). 